\title{
A Review on Data Security in Cloud Computing
}

\author{
Aized Amin Soofi, M. Irfan Khan \\ College of Computer Science and Information \\ studies \\ Government College University \\ Faisalabad, Pakistan
}

\author{
Fazal-e-Amin \\ Department of Software Engineering \\ College of Computer and Information Sciences \\ King Saud University, Riyadh, KSA
}

\begin{abstract}
Cloud computing is an Internet-based computing and next stage in evolution of the internet. It has received significant attention in recent years but security issue is one of the major inhibitor in decreasing the growth of cloud computing. It essentially shifts the user data and application software to large datacenters i.e, cloud, which is remotely located, at which user does not have any control and the management of data may not be completely secure. However, this sole feature of the cloud computing introduce many security challenges which need to be resolved and understood clearly. One of the most important and leading is security issue that needs to be addressed. Data Security concerns arising because both user data and program are located in provider premises. In this study, an attempt is made to review the research in this field. The results of review are categorized on the basis of type of approach and the type of validation used to validate the approach.
\end{abstract}

\section{Keywords}

Data security, cloud data concealment, cloud security, review

\section{INTRODUCTION}

Cloud computing is an emerging technology which recently has drawn significant attention from both industry and academia. It provides services over the internet, by using cloud computing user can utilize the online services of different software instead of purchasing or installing them on their own computers. According to the National Institute of Standard and Technology (NIST) definition, cloud computing can be defined as a paradigm for enabling useful, on-demand network access to a shared pool of configurable computing resources [1]. According to Gartner [2] cloud computing can be defined as a style of computing that delivered IT capabilities 'as a service' to end users through internet.

According to recent survey by International Data Group (IDG) enterprise, the top three challenges to implementing a successful cloud strategy in enterprise vary significantly between IT and line-of-business (LOB). For IT, concerns regarding security is (66\%) and $42 \%$ of cloud-based projects are eventually brought back in-house, with security concerns (65\%) [3]. A survey conducted by International Data Corporation (IDC) in 2011 declares that $47 \%$ IT executives were concerned about a security threats in cloud computing [4]. In survey conducted by Cisco's CloudWatch 2011 report for the U.K. (research conducted by Loudhouse) $76 \%$ of respondents cited security and privacy a top obstacle to cloud adoption [5].

Data security is a major concern for users who want to use cloud computing. This technology needs proper security principles and mechanisms to eliminate users concerns. Most of the cloud services users have concerns about their private data that it may be used for other purposes or sent to other cloud service providers [6]. The user data that need to be protected includes four parts [7] which are: (i) usage data; information collected from computer devices (ii) sensitive information; information on health, bank account etc. (iii) Personally identifiable information; information that could be used to identify the individual (iv) Unique device identities; information that might be uniquely traceable e.g. IP addresses, unique hardware identities etc.

The European Network and Information Security Agency (ENISA) identified thirty-five risks and these risks are divided into four categories: legal risk, policy and organizational risks, technical risks and risks that are not specific to cloud [8]. From these risks, the ENISA identified eight most important risks. Out of which five risks concerns directly or indirectly related to the data confidentiality. These risks include isolation failure, data protection, management interface compromise, insecure data deletion and malicious insider. Similarly, The Cloud Security Alliance (CSA) identifies the thirteen kind of risks related to the cloud computing [9]. Out of these thirteen risks CSA declares seven most important risks [10]. Five of these seven risks are directly or indirectly related to the data confidentiality which includes: account service, traffic hijacking, insecure application programming interfaces, data loss/leakage and malicious insiders.

Different countries, IT companies, and the relevant departments have carried out the research on cloud computing security technology to expand the security standards of cloud computing. Existing security technology reflected in six aspects[11,12] which include: data privacy protection, trusted access control, cloud resource access control, retrieve and process of cipher text, proof of existence and usability of data and trusted cloud computing. To enhance the data security the data can be converted into cipher text but this may cause to lose many features when data is converted into cipher text.

There are two widely used methods to retrieve the cipher text. First, there is a safety index-based approach which establishes a secure cipher text key words indexed by checking the existence of key words [13]. Second, there is a cipher text scanning-based approach which confirms the existence of key words by matching each word in cipher text [14]. [15] Lists the top ten obstacles in the popularity of cloud computing. The data security and storage issues is discussed in this article and it also analyzes the main reasons of data security issue, possible solutions of this issues and some future development of cloud computing are also discussed. [16] Explains the seven phase of data life cycle in cloud computing that also 
need security to get user trust these phase include; generation, transfer, use, share, storage, archival and destruction. The aim of cloud computing is to provide better consumption of resources and reduce the work load from user end but it suffers with security threats [17]. The complexity of security in complete cloud computing environment is shown in fig 1.

In figure 1the lower layer indicates the deployment models of cloud computing namely private cloud, community cloud, public cloud and hybrid cloud. The layer just above the deployment model represents the services delivery model of cloud computing. These service delivery models exhibit the certain characteristics that are shown in the top layer. These fundamental elements need security with respect to the characteristics of selected deployment model. Some of fundamental security challenges are shown in the vertical layer given in figure 1 .

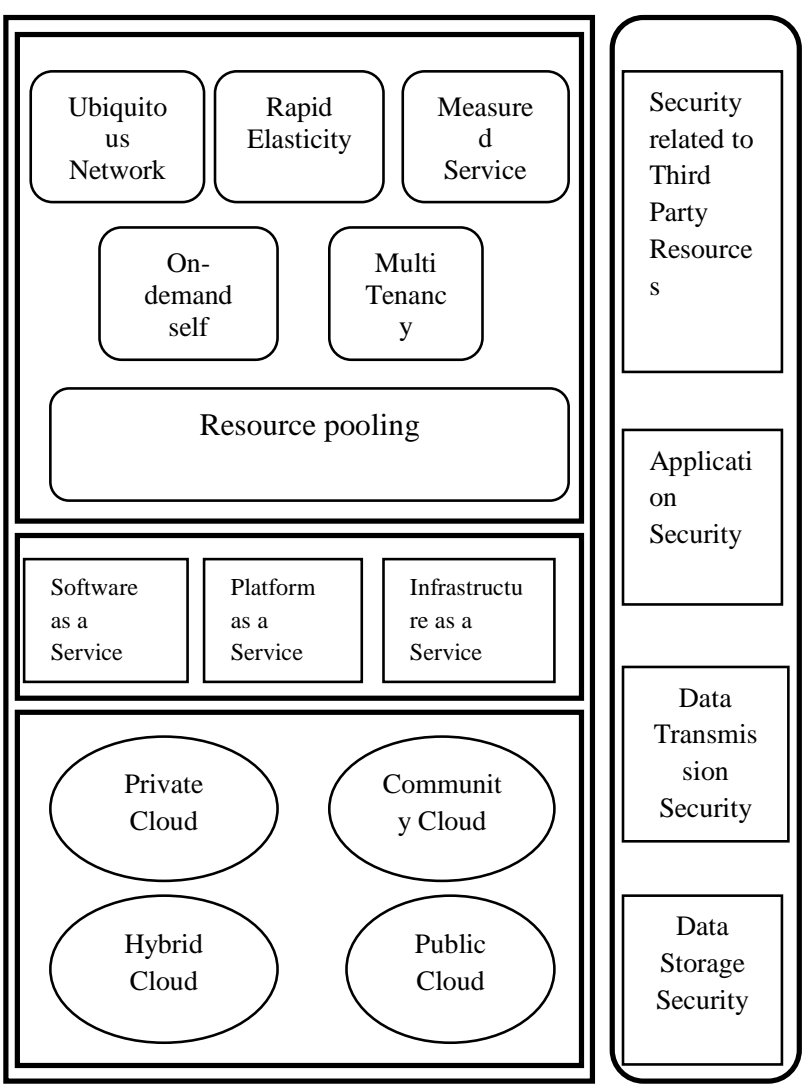

Fig.1 Complexity of security in cloud environment [21]

\section{CLASSIFICATION OF CLOUD COMPUTING}

The main attributes of cloud computing are Multi-tenancy, massive scalability, elasticity, pay as you go and selfprovisioning of resources [18]. The services model of cloud computing is divided into three categories (1) IaaS (infrastructure as a service) provides the use of virtual computer infrastructure environment, online storage, hardware, servers and networking components; (2) PaaS (plat form as a service) provides platform for developing applications by using different programming languages; (3) SaaS (software as a service) enables the user to access online applications and software that are hosted by the service providers. The deployment model of cloud computing include (1) public cloud, that owned by service provider and its resources are rented or sold to the public (2) private cloud, that is owned or rented by an organization (3) community cloud, that is similar to private cloud but cloud resources is shared among number of closed community (4) hybrid cloud, exhibits the property of two or more deployment models [19]. Figure 2 shows the NIST definition framework for cloud computing.

\begin{tabular}{|c|c|c|c|}
\hline \multicolumn{4}{|l|}{ Deployment } \\
\hline $\begin{array}{l}\text { Service } \\
\text { Models }\end{array}$ & $\begin{array}{l}\text { Software as a } \\
\text { Service (SaaS) }\end{array}$ & $\begin{array}{l}\text { Platform as a } \\
\text { Service (PaaS) }\end{array}$ & $\begin{array}{l}\text { Infrastructure as a } \\
\text { Service (laaS) }\end{array}$ \\
\hline \multirow{3}{*}{$\begin{array}{l}\text { Essential } \\
\text { Characteristics }\end{array}$} & \multicolumn{3}{|c|}{ On Demand Self-Service } \\
\hline & Broad Network Access & & id Elasticity \\
\hline & Resource Pooling & & ured Service \\
\hline \multirow{4}{*}{$\begin{array}{l}\text { Common } \\
\text { Characteristics }\end{array}$} & Massive Scale & & ent Computing \\
\hline & Homogeneity & Geo & ohic Distribution \\
\hline & Virtualization & & Orientation \\
\hline & Low Cost Software & & nced Security \\
\hline
\end{tabular}

Fig2: NIST cloud definition Frame work [20]

In this research work we focused on the data security issue in cloud computing environment. Public cloud deployment model mostly suffers from the risk of data security. On the other hand, in SaaS delivery model client is dependent on service provider for proper security measures. The provider must implement some strict security measures to keep multiple users from seeing each other's data and gain the trust of users. Recent reviews on security issues in cloud computing are presented in $[21,22,23]$ but these reviews are limited and not focused on detail study of data security issue. Neither of them adopts a proper literature review process. In our study we focused in details study on data security issue by adopting a proper systematic literature review process.

\section{METHODOLOGY}

Empirical studies are now being undertaken more frequently, as a means of examining a broad range of phenomenon in computer field. A systematic literature review presented in [24] is followed in this research work to conduct the review. The review process is shown in figure 3. A systematic literature review endeavor to provide a comprehensive review of current literature relevant to a specified research questions.

Many researchers contribute their efforts in the field of software engineering/computer science by adopting [24] systematic literature review process such as in $[25,26]$ systematic literature review process is adopted for the review of aspect oriented implementation of software product lines components and software component reusability assessment approaches. 


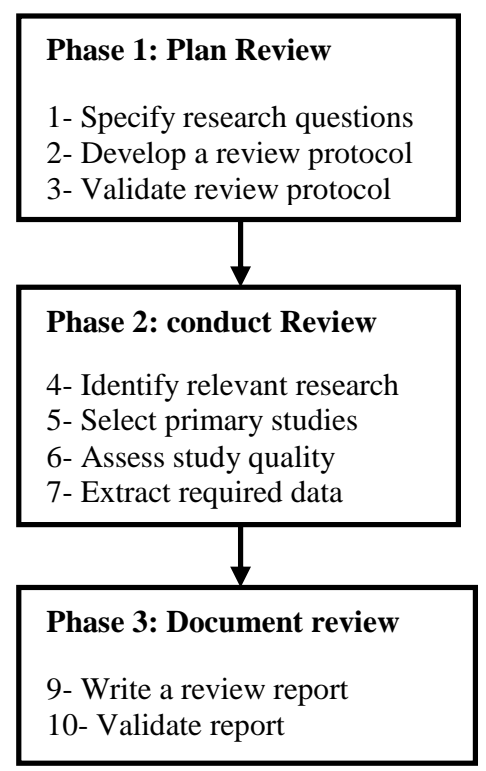

Fig 3: Adapted review process from [24]

The review process has three phases that consist of ten sub activities. In first phase of review the following questions are posed:

Question 1: What approaches have been introduced to ensure data security in cloud computing?

Question 2: How the approaches have been validated?

The questions are formulated during the first sub activity of phase 1, a review protocol was developed. The review protocol includes the sources, time period under review and key words used. This protocol is reviewed and validated after making some changes by researchers.

The final review protocol is shown in table 1 . The sources used for this review include science direct, IEEE xplorer, Google scholar, Scopus, ACM portal digital library. Additionally we have looked at JCMS, IJSI journals. The research focuses on the year's 2007 to 2014 .

Table 1. Review protocol

\begin{tabular}{|l|l|l|}
\hline Year & sources & Key words \\
\hline 2007-2014 & $\begin{array}{l}\text { IEEE Xplore, } \\
\text { science direct, } \\
\text { Scopus, Google } \\
\text { scholar, ACM } \\
\text { portal digital }\end{array}$ & $\begin{array}{l}\text { Cloud computing, } \\
\text { cloud computing } \\
\text { security, data } \\
\text { security/data } \\
\text { concealment, cloud } \\
\text { dibrary, IJERA, } \\
\text { data security, cloud } \\
\text { data storage }\end{array}$ \\
\hline
\end{tabular}

In the second phase of review, the search is performed by using different queries related to data security in cloud computing environment. The initial collection of research papers was based on the key words in Table 1 in the papers keywords and abstract. The quality criteria set to assess the studies was to include papers in the review if it contains a model, an experiment, a framework, or a guideline. The required data was extracted from the papers to answer the questions posed above.

Another step in the search process was performed by searching the related work area of the selected papers to boost the review strength by confirming that no valuable reference is missed during the search process. The collected data was synthesized to exhibit complete results. Finally, in the third phase of the review process, the review report was written and validated.

\section{RESULTS}

The results of the review are presented in this section. A year wise result representation is presented in Table 2 and frequency of papers with respect to sources is shown in Fig 4. The results are characterized with respect to the questions posed earlier.

Table 2 year wise search results

\begin{tabular}{|l|l|}
\hline Year & No. of papers \\
\hline 2007 & 0 \\
\hline 2008 & 1 \\
\hline 2009 & 1 \\
\hline 2010 & 5 \\
\hline 2011 & 5 \\
\hline 2012 & 8 \\
\hline 2013 & 9 \\
\hline 2014 & 2 \\
\hline Total & 31 \\
\hline
\end{tabular}

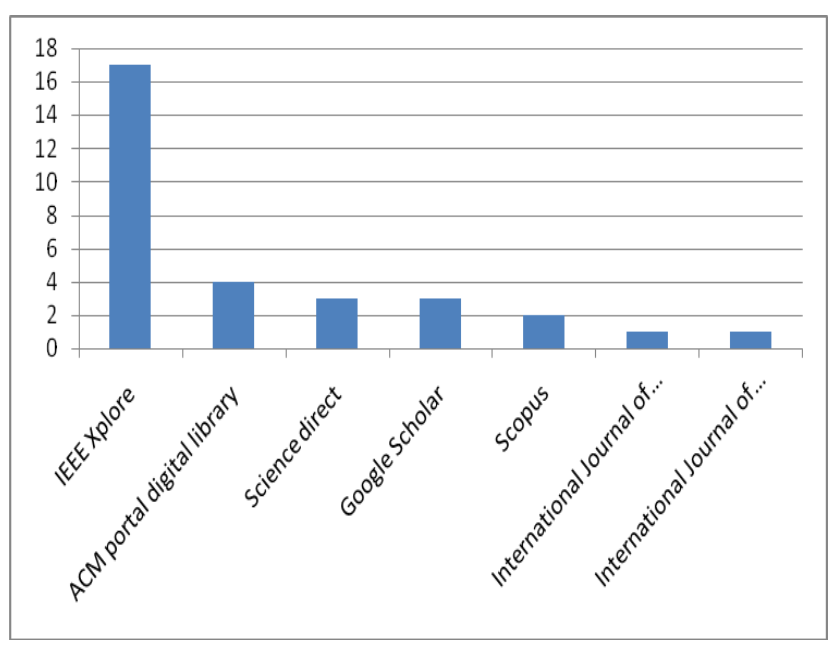

Fig4: Frequency of papers w.r.t to sources

\section{QUESTION-1: WHAT APPROACHES HAVE BEEN INTRODUCED TO ENSURE DATA SECURITY IN CLOUD COMPUTING?}

The result of review (figure 5) show the proposed approaches for the data security in cloud computing. These results are categorized into: (1) Encryption, where the plain text is 
converted into cipher text by using some encryption algorithms; (2) Homomorphic token. A technique ensures that we do not need to decrypt the key for data checking instead we can directly compare with encrypted token; (3) Guidelines. Some of the studies have outlined some guidelines to ensure the data security in cloud; (4) Harmonizing scheme. Building a data repository; (5) data concealment component; (6) token; (7) Framework; (8) stripping algorithm.

The categories wise results are summarized in Table 3.

Table 3 category wise results of question1

\begin{tabular}{|l|l|l|}
\hline Question & category & No. of papers \\
\hline $\begin{array}{l}\text { What approaches } \\
\text { have been } \\
\text { introduced to ensure } \\
\text { data security in } \\
\text { cloud computing? }\end{array}$ & Encryption & 14 \\
& $\begin{array}{l}\text { Sobol sequence } \\
\text { Harmonizing scheme }\end{array}$ & 1 \\
& $\begin{array}{l}\text { Data concealment } \\
\text { component } \\
\text { Framework }\end{array}$ & 1 \\
& $\begin{array}{l}\text { Stripping algorithm } \\
\text { Total }\end{array}$ & 1 \\
\hline
\end{tabular}

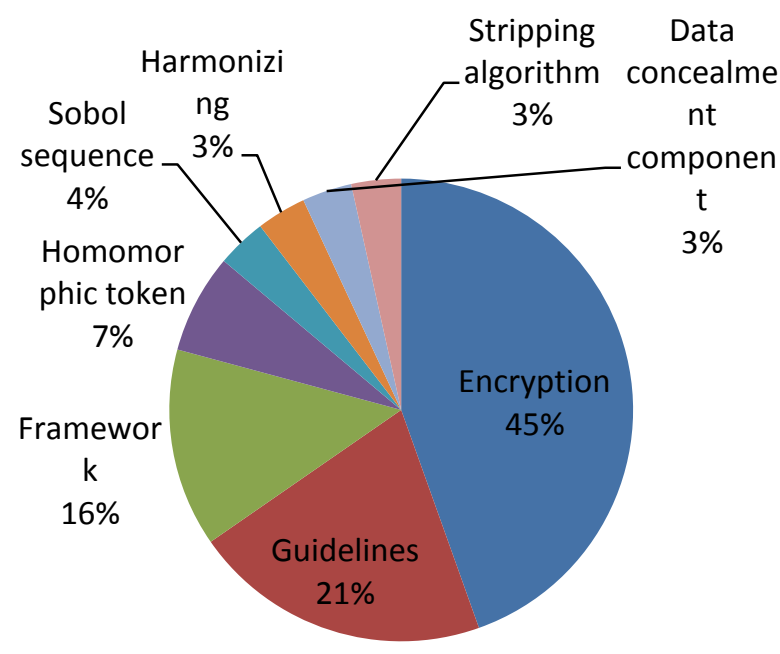
Fig 5: Proposed approaches to ensure data
security

\subsection{Encryption}

The results show that most common approach was encryption (45\%) to assure the data security in cloud. In [27] a digital signature with RSA algorithm scheme is proposed to ensure the data security in cloud. In which software used to crunch down the data documents into few lines by using "hashing algorithm". These lines are called message digest then software encrypts the message digest with his private key to produce the digital signature. Digital signature will be decrypted into message digest by the software with own private key and public key of sender.

In [28] playfair and vigenere cipher techniques were merged with structural aspects of Simplified Data Encryption Standard (SDES) and Data Encryption Standard (DES). In which 64 bit block size of plain text is taken which is fixed and this 64 bit plain text is divided into two halves by using the "black box" the right half have 2 bits whereas left half has 6 bits, then these 6 bits are feed into "superior function" block where these 6 bits are further separated in two halves where first two bits represent the rows and last four bits represent the column by identifying the rows and column the corresponding value can be selected. Then this function is apllied to all 8 octets of the output of vigenere block the resultant of black box is again of 64 bits then these bits are further divided into 4 new octants similarly right 4 bits are unified to formulate right halves. Finally left and right halves are XOR-ed to obtain left half of this arrangement. This process is repeated three times.

In [29] RSA algorithm used to encrypt the data and Bilinear Diffie-Hellman to insure the security while exchanging the keys. In proposed method a message header is added in front of each data packet for direct and safe communication between client and cloud without any third party server. When user sends the request to the cloud server for data storage then cloud server creates the user public key, private key and user identification in certain server. Two tasks performed at user end before sending the file to cloud, first add message header to the data and secondly encrypt data including message header by using secret key. When user request for data to the cloud server then it will check the message header of received data and pick up the Unique Identification for Server in cloud (SID) information. If SID information is found it will respond the user request otherwise request will be discarded.

In [30], a technique is introduced to ensure the availability, integrity and confidentiality of data in cloud by using Secure Socket Layer (SSL) 128 bit encryption that can also be raised to 256 bit encryption. The user who wishes to access the data from cloud is strictly required to provide valid user identity and password before access is given to the encrypted data. In [31], user send the data to the cloud then cloud service provider generate a key and encrypts the user data by using RSA algorithm and stored the data into its data centre. When user request the data from cloud then cloud service provider verify the authenticity of the user and give the encrypted data to the user that can be decrypted by calculating the private key.

In [32], a three layered data security model is presented in which each layer performs different task to make the data secure in cloud. First layer is responsible for authentication, second layer performs the duty of data encryption and third layer performs the functionality of data recovery. In [33], RC5 algorithm is implemented to secure the data in cloud. An encrypted data is transmitted even if the data is stolen there will be no corresponding key to decrypt the data. In [34] Role Base Encryption (RBE) technique is proposed to secure the data in cloud and role base access control (RBAC) cloud architecture was also proposed which allows organizations to store data securely in public cloud, while maintaining the secret information of organization's structure in private cloud. 
In [35], four authorities are defined i.e, data owner, data consumer, cloud server and $\mathrm{N}$ attribute authorities where attribute authorities sets was divided into $\mathrm{N}$ disjoint sets with respect to the category. The data owner gets the public key from any one of the authority and encrypt the data before sending it to the cloud server. When data is requested the authorities will create private key and send it to the data consumer and consumer will be able to download the file only if he get verified by cloud server. In [36], two types of secure cloud computing are proposed one require trusted third party and other does not. These types use Elliptic Curve DiffieHellman (ECDH) and symmetric bivariate polynomial based secret sharing to ensure the data security in cloud environment.

In [37], location based encryption technique by using user location and geographical position was introduced. In which a geo encryption algorithm was implemented on the cloud and user computer and the data was labeled with the company name or person who work in the company. When the data is required then in the cloud similar label will be searched and retrieved and the information corresponding to the label will be retrieved. In [38], a technique is proposed by using digital signature and Diffie Hellman key exchange in combination with Advanced Encryption Standard encryption algorithm to protect the confidentiality of data stored in cloud. This scheme is referred as three way mechanism because it provides authentication, data security and verification at the same time.

\subsection{Guidelines}

The result of our review shows that $21 \%$ of studies use guidelines to ensure the security of data in cloud. In [39], guidelines are provided for data security in cloud by introducing new cloud system architecture approach which has three features i.e., separation of software service providers and infrastructure service providers, hiding information about owner of data and data obfuscation. In [40], agents method is introduce to ensure the data security in cloud architecture. In which three agents namely file agent, authentication agent and key managing agent was used for data security.

In [41], guidelines about six key data technologies are provided which are: data privacy protection, proof of existence and usability of data, trusted access control, retrieve and process of cipher text, cloud resource access control and trusted cloud computing. In [42], guidelines are provided by giving the meta analysis of four different encryption algorithms that are also helpful to selecting the best algorithms according to need.

\subsection{Framework}

The framework approach represents $14 \%$ of the results. In [43], a framework is provided; known as TrustCloud, in which data centric and detective approach is propose to increase the security of data with the objectives to encourage the adoption of file-centric and data- centric logging mechanism to increase the security and confidentiality of data in cloud computing. In [44], a framework is provided by building a multi-tenant system. In which developed solution is divided into three layers i.e. presentation layer, business logic layer and data access layer. These layers provide very high security to user data.

In [45], a framework is provided that consists of protocol named SecCloud, which is a first protocol spanning secure storage and secure computation in cloud environment by designated verifier signature, batch authentication and probabilistic sampling procedures. In [46], proposed framework is consist of three steps, in first step precaution is made against semi-honest cloud service provider by indexing data and its metadata to ensure complete data privacy. In second step multi user private keyword searchable encryption is performed on encrypted data to keep searches and resulting files secrecy from cloud service provider. Final step make the use of policy in order to support data sharing between users by using metadata and encryption scheme.

\subsection{Homomorphic Token}

The homomorphic token scheme represents the $7 \%$ of the results. In [47], homomorphic token scheme is introduced to ensure the data security. The proposed scheme utilizes homomorphic token with distributed verification of erasurecoded data. It supports secure and efficient dynamic operation on data block including data delete, update and append. A model proposed in [48] by utilizing homomorphic token scheme with token pre- computation algorithm to achieves the integration of storage correctness insurance and identification of misbehaving server(s).

\subsection{Stripping algorithm, data concealment component, harmonizing and token scheme}

Stripping algorithm, data concealment component, and harmonizing and token scheme each represent $3 \%$ of the results. In [49], stripping algorithm is used to secure the picture data in cloud, the approach is consist of three modules which are image analysis, data separation and data distribution. [50] Proposed a design of data concealment component that composed of three sub components: the prediction component, data generator and data marking to secure the data in cloud. The Evaluation of this component shows the successful conceal data of legitimate users and protect them against potential attacks.

A privacy preserving repository presented in [51], this repository was basically concentrated on the harmonizing operations to achieve data confidentiality while still keeping the harmonizing relations intact in the cloud. This proposed scheme make data owner enables to assign most of computation intensive tasks to cloud servers without disclosing data contents. [52] Proposed an effective and flexible distribution verification protocol to address data security in cloud computing. This protocol utilizes token precomputation using sobol sequence to verify the integrity of erasure coded data instead of pseudorandom data. The proposed model consist of three phases that are: file distribution, token pre-computation and challenge response protocol.

\section{QUESTION-2: HOW THE APPROACHES HAVE BEEN VALIDATED?}

The results related to the second question are presented here. Figure 6 shows the result of review regarding the procedures adopted for validation. The categories are: (1) Experiment, where an experiment is carried out to validate the results; (2) Comparative analysis, where the results of proposed scheme is compared to other schemes to validate the results; (3) Test bed is used to validate the proposed approach; (4) Statistical analysis, where the results are analyzed by using some statistical technique; (5) Meta analysis is used to validate the 
results; (6) Performance analysis, where the performance of proposed approach is analyzed by different methods; (7) Some of the proposed approaches have not performed any validation. The category wise detail is presented in table IV and fig 6 shows the type of validation in percentage. Let us explain the term validation. It refers to any kind of empirical method used as a proof, apart from the demonstration/ application of the proposed approach.

Table 4 categories wise results of question 2

\begin{tabular}{|c|c|c|}
\hline Question & category & No. of papers \\
\hline $\begin{array}{l}\text { How the approaches } \\
\text { have been validated? }\end{array}$ & $\begin{array}{l}\text { Experiment } \\
\text { No Validation } \\
\text { Comparative } \\
\text { Analysis } \\
\text { Meta Analysis } \\
\text { Test Bed } \\
\text { Statistical Analysis } \\
\text { Performance } \\
\text { Analysis } \\
\text { Total }\end{array}$ & $\begin{array}{l}10 \\
13 \\
3 \\
1 \\
1 \\
1 \\
2 \\
31\end{array}$ \\
\hline
\end{tabular}

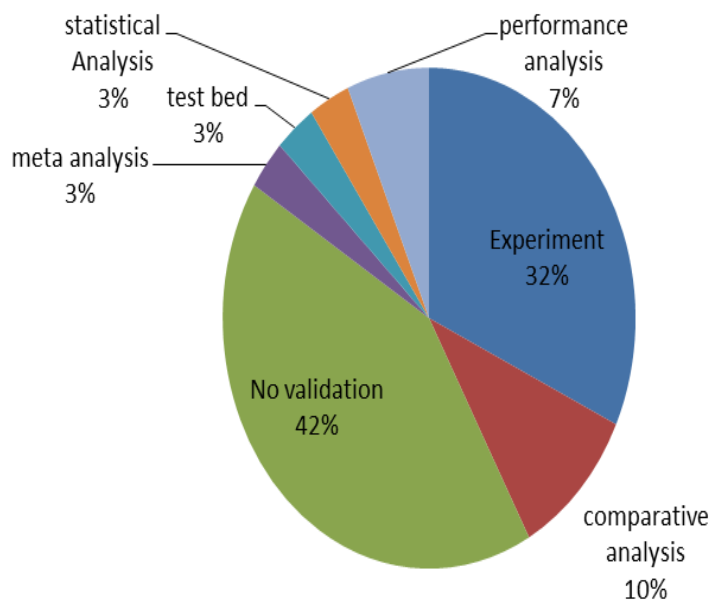

Fig 6: Type of validation

The results of the question regarding validation of proposed approaches show that $47 \%$ of the selected papers proposed approach to secure data in the cloud environment but provide no validation of the proposed approach.

\subsection{Experimental Approach}

Experiments are used to validate the proposed approach in $32 \%$ of the selected papers. In [30], experiment was performed to test the validity of proposed model by using cloud simulator named Hadoop. It shows the status of security after implementing three security parameters which are; Message Authentication Code, classification of data, index and encryption technique. In [33] Aneka 2.0 software is used in cloud environment to validate the results obtaining by the implementation of RC5 algorithm and then compare these results with Amazon S3 service. Aneka allows building and managing an interconnected network by using Microsoft .NET frameworks on these networks.

In [34] proposed architecture is implemented in Java and results show that cipher text size is linearly proportional to the size of the plaintext and the efficiency of encryption and decryption is very good. Results also show that the size of the decryption key is 48 bytes which is convenient for the users. In [39] cloud service is implemented using C\# Microsoft .NET framework for collaborative online documentation. The experimental results shows that service response time increases linearly as the size of the input text increases and data obfus-cation and de-obfuscation do not cause much overhead, hence proposed approach showed realistic performance. In [50] PHP language was used for the experiment in which performance test is conducted for three phases that are data generation, data marking and data extraction. During the performance test impact of component on data generation was also observed.

\subsection{Comparative Analysis}

Comparative analysis as the form of validation is employed in $10 \%$ of the selected studies in which results of proposed scheme is compared to other schemes to validate the results. In [53] comparative analysis is conducted to validate the results by considering following variables granularity, key management, meta data management, level of concealment, degree of distribution and level of implementation. In [36] comparative analysis is made between data Privacy by Authentication and Secret Sharing (PASS) and proposed technique that used trusted third party and non trusted third party. In [28], the proposed encryption technique is compared with DES, SDES, Playfair and Vigenere encryption technique to validate the proposed approach results.

\subsection{Performance Analysis}

Performance analysis is used to validate the proposed approach in $7 \%$ of selected papers. In [47], performance analysis is performed in terms of security and efficiency to show that the results are validated and proposed scheme is highly efficient and flexible against Byzantine failure and malicious data modification attacks.

\subsection{Statistical Analysis}

Statistical analysis, meta analysis and test bed as the form of validation are employed in $3 \%$ of the selected studies. In [32] NIST statistical test are used to validate the results by selecting eight modern encryption algorithms. In [42], meta analysis of four different security algorithms which are; AES, RSA, Blow fish and DES are presented in term of platform, key size, key used, scalability, initial vector size, security, data encryption capacity, authentication type, memory usage and execution time to validate the results. In [45], test bed is developed and tested for the validation of results.

\section{CONCLUSIONS AND FUTURE DIRECTIONS}

There are many benefits of using cloud computing such as cost efficiency, quick deployment, improved accessibility etc. However, there are yet many practical problems which have to be solved. The data confidentiality is one of them. Many 
researchers contributed their efforts to minimize the data security issue in this domain with different solutions that described in this work. A literature review of the works in the area of cloud computing data security is conducted and the results of review are presented in this paper. The results show that the majority of approaches are based on encryption (45\%) out of which $71 \%$ encryption techniques results are validated. $67 \%$ of encryption techniques used experimentation to validate the results. These results point towards the fact that most of researchers show their interest in encryption technique to enhance the security of data in cloud computing environment. The results also reveals the fact of lack of validation in proposed approaches as $42 \%$ of the studies provide no validation of the results out of which $67 \%$ are guidelines. Only few studies have used statistical analysis for validation. This area (validation) needs the attention of the research community to gain the trust and confidence of cloud computing users.

Although our review has explored the field, further studies are needed to confirm the obtained results. Future work includes the extension of this review by including more sources (conferences, journals and workshops) and questions. A future plan is to explore the other security issues in the cloud computing environment and we are also aiming to design a security model using some encryption techniques for data concealment in cloud computing.

\section{REFERENCES}

[1] NIST SP 800-145, "A NIST definition of cloud computing", [online] 2012 , http://csrc.nist.gov/publications/drafts/800145/Draft-SP- 800-145_cloud-definition.pdf (Accessed: 23 December 2013)

[2] Gartner,"What you need to know about cloud computing security and compliance"(Heiser J), [online] 2009 https://www.gartner.com/doc/1071415/need-knowcloud- computing- Security (Accessed 23 December 2013).

[3] IDG Cloud Computing Survey: "Security, Integration Challenge Growth", [online] http://www.forbes.com/sites/louiscolumbus/2013/08 /13/idg- cloud-computing-survey- (Accessed: 28 December 2013).

[4] Ricadela, "Cloud security is looking overcast"[online]

http://www.businessweek.com/magazine/cloudsecurity- is-lookin g-overcast-09012011.html. (Accessd: 29December 2013).

[5] Nguyen, "Only seven percent of UK it services in the cloud, says survey,Computerworld"[online] http://www.itworld.com/ cloudcomputing/200657/only- seven-percent-uk-itservices-cloud-says- surveyS. (Accessed: 29 December 2013).

[6] Elahi, T., \& Pearson, S. (2007). Privacy Assurance: Bridging the Gap Between Preference and Practice. In C. Lambrinoudakis, G. Pernul \& A. Tjoa (Eds.), Trust, Privacy and Security in Digital Business (Vol. 4657, pp. 65-74): Springer Berlin Heidelberg.

[7] Siani Pearson, "Taking Account of Privacy when Designing Cloud Computing Services,"
CLOUD’09, May 23, 2009, Vancouver, Canada, pp. 44-52.

[8] European Network and Information Security Agency (ENISA)"Benefits, risks and recommendations for information security"[online] http://www.enisa.europa.eu/activities/riskmanageme nt/files/ deliverables/cloud-computing- riskassessment. (Accessed: 28.December 2013).

[9] Cloud Security Alliance, "Security Guidance for Critical Areas of Focus in Cloud Computing"[online] https://cloudsecurityalliance.org/csaguide.pdf (Accessed 26 December2013)

[10] J. Archer et al., "Top Threats to Cloud Computing," in Cloud Security Alliance [online] https://cloudsecurityalliance.org/topthreats/csathreat s.v1.0.pdf (Accessed: 26 December 2013).

[11] Crampton, J., Martin, K., \& Wild, P. (2006, 0-0 0). On key assignment for hierarchical access control. Paper presented at the Computer Security Foundations Workshop, 2006. 19th IEEE.

[12] D.Feng, et al. "Study on cloud computing security." Journal of Software 22.1 (2011): pp.71-83.

[13] R. Chow, et al., "Controlling data in the cloud: Outsourcing computation without outsourcing control," presented at the Proceedings of the 2009 ACM workshop on Cloud computing security, Chicago, Illinois, USA, 2009.

[14] S. Dawn Xiaoding, et al., "Practical techniques for searches on encrypted data," in Security and Privacy, 2000. S\&P 2000. Proceedings. 2000 IEEE Symposium on, 2000, pp. 44-55.

[15] Michael Annbrust etc.,Above the Clouds: A Berkeley View of Cloud Computing, http: //eecs.berkeley.edu/Pubs/TechRpts/2009 /EECS 2009-28.pdf:2009.2 .

[16] Deyan, C., \& Hong, Z. (2012, 23-25 March 2012). Data Security and Privacy Protection Issues in Cloud Computing. Paper presented at the Computer Science and Electronics Engineering (ICCSEE), 2012 International Conference on

[17] Seccombe, A., Hutton, A., Meisel, A., Windel, A., Mohammed, A., \& Licciardi, A. (2009). Security guidance for critical areas of focus in cloud computing, v2. 1. Cloud Security Alliance.

[18] T. Mather and S. Latif, "Cloud Security and

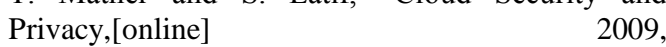
http://www.slideshare.net/USFstudent1980/cloudcomputing security-concerns (Accessed: 4 September 2013)

[19] IBM, "what is cloud computing" [online] http://www.ibm.com/cloud- computing/in/en/whatis-cloud-computing.html (Accessed: 14 December 2013)

[20] Mell Peter and Grance Tim, "Effectively and securely using the cloud computing paradigm" [online] 2011, http://csrc.nist.gov/groups/SNS/cloud computing/cloudcomputing-v26.ppt (Accessed 18 August 2013). 
[21] Subashini, S., \& Kavitha, V. (2011). A survey on security issues in service delivery models of cloud computing. Journal of Network and Computer Applications, 34(1), 1- 11.

[22] Sarwar, A., \& Khan, M. N. (2013). A Review of Trust Aspects in Cloud Computing Security. International Journal of Cloud Computing and Services Science (IJ-CLOSER), 2(2), 116-122.

[23] Sun, D., Chang, G., Sun, L., \& Wang, X. (2011). Surveying and Analyzing Security, Privacy and Trust Issues in Cloud Computing Environments. Procedia Engineering, 15(0), 2852-2856.

[24] Brereton, P., Kitchenham, B. A., Budgen, D., Turner, M., \& Khalil, M. (2007). Lessons from applying the systematic literature review process within the software engineering domain. Journal of Systems and Software, 80(4), 571-583.

[25] Fazal-e-Amin, A. K. M., \& Oxley, A. (2010). A review on aspect oriented implementation of software product lines components. Information Technology Journal, 9(6), 1262-1269.

[26] Fazal-e-Amin, A. K. M., \& Oxley, A. (2011). A Review of Software Component Reusability Assessment Approaches. Research Journal of Information Technology, 3(1), 1-11.

[27] Somani, U., Lakhani, K., \& Mundra, M. (2010, 2830 Oct. 2010). Implementing digital signature with RSA ncryption algorithm to enhance the Data Security of cloud in Cloud Computing. Paper presented at the Parallel Distributed and Grid Computing (PDGC), 2010 1st International Conference on.

[28] Vamsee k and sriram r,(2011) "Data Security in Cloud Computing,"in Journal of Computer and Mathematical Sciences Vol. 2, pp.1-169.

[29] Shuai, H., \& Jianchuan, X. (2011, 15-17 Sept. 2011). Ensuring data storage security through a novel third party auditor scheme in cloud computing. Paper presented at the Cloud Computing and Intelligence Systems (CCIS), 2011 IEEE International Conference on.

[30] Sood, S. K. (2012). A combined approach to ensure data security in cloud computing. Journal of Network and Computer Applications, 35(6), 18311838 .

[31] Parsi Kalpana \& Sudha Singaraju (2012).Data Security in Cloud Computing using RSA Algorithm. International Journal of Research in Computer and Communication technology( IJRCCT), vol 1, Issue 4.

[32] Mohamed, E. M., Abdelkader, H. S., \& El-Etriby, S. (2012,14-16 May 2012). Enhanced data security model for cloud computing. Paper presented at the Informatics and Systems (INFOS), 2012 8th International Conference on.

[33] Singh, J., Kumar, B., \& Khatri, A. (2012, 6-8 Dec. 2012). Improving stored data security in Cloud using Rc5 algorithm. Paper presented at the Engineering (NUiCONE), 2012 Nirma University International Conference on.
[34] Lan, Z., Varadharajan, V., \& Hitchens, M. (2013). Achieving Secure Role-Based Access Control on Encrypted Data in Cloud Storage. Information Forensics and Security, IEEE Transactions on, 8(12), 1947-1960.

[35] Taeho, J., Xiang-Yang, L., Zhiguo, W., \& Meng, W. (2013, 14-19 April 2013). Privacy preserving cloud data access with multi-authorities. Paper presented at the INFOCOM, 2013 Proceedings IEEE.

[36] Ching-Nung, Y., \& Jia-Bin, L. (2013, 2-5 July 2013). Protecting Data Privacy and Security for Cloud Computing Based on Secret Sharing. Paper presented at the Biometrics and Security Technologies (ISBAST), 2013 International Symposium on.

[37] Abolghasemi, M. S., Sefidab, M. M., \& Atani, R. E. (2013, 22-25 Aug. 2013). Using location based encryption to improve the security of data access in cloud computing. Paper presented at the Advances in Computing, Communications and Informatics (ICACCI), 2013 International Conference on.

[38] Rewagad, P., \& Pawar, Y. (2013, 6-8 April 2013). Use of digital Signature with Diffie Hellman Key Exchange and AES Encryption Algorithm to Enhance Data Security in Cloud Computing. Paper presented at the Communication Systems and Network Technologies CSNT), 2013 International Conference on.

[39] Yau, S. S., \& An, H. G. (2010). Protection of users' data confidentiality in cloud computing. Paper presented at the Proceedings of the Second AsiaPacific Symposium on Internetware.

[40] Feng-qing, Z., \& Dian-Yuan, H. (2012, 24-26 Aug. 2012). Applying agents to the data security in cloud computing. Paper presented at the Computer Science and Information Processing (CSIP), 2012 International Conference on.

[41] Zhongbin, T., Xiaoling, W., Li, J., Xin, Z., \& Wenhui, M. (2012, 27-30 May 2012). Study on Data Security of Cloud Computing. Paper presented at the Engineering and Technology (S-CET), 2012 Spring Congress on.

[42] Rachna, A., and Anshu, P.(Jul-Aug 2013). Secure User Data in Cloud Computing Using Encryption Algorithms in International Journal of Engineering Research and Applications (IJERA), 3(4),19221926.

[43] Ko, R. K. L., Kirchberg, M., \& Bu Sung, L. (2011, 3-5 Aug. 2011). From system-centric to data-centric logging Accountability, trust \&amp; security in cloud computing. Paper presented at the Defense Science Research Conference and Expo (DSR), 2011.

[44] Gawali, M. B., \& Wagh, R. B. (2012, 6-8 Dec. 2012). Enhancement for data security in cloud computing environment. Paper presented at the Engineering (NUiCONE), 2012 Nirma University International Conference on.

[45] Wei, L., Zhu, H., Cao, Z., Dong, X., Jia, W., Chen, Y., et al. (2014). Security and privacy for storage 
and computation in cloud computing. Information Sciences, 258(0), 371-386.

[46] Rashid, F., Miri, A., \& Woungang, I. (2013, June 28 2013-July 3 2013). Secure Enterprise Data Deduplication in the Cloud. Paper presented at the Cloud Computing (CLOUD), 2013 IEEE Sixth International Conference on.

[47] Cong, W., Qian, W., Kui, R., \& Wenjing, L. (2009, 13-15 July 2009). Ensuring data storage security in Cloud Computing. Paper presented at the Quality of Service, 2009. IWQoS. 17th International Workshop on.

[48] Tribhuwan, M. R., Bhuyar, V. A., \& Pirzade, S. (2010, 16-17 Oct. 2010). Ensuring Data Storage Security in Cloud Computing through Two-Way Handshake Based on Token Management. Paper presented at the Advances in Recent Technologies in Communication and Computing (ARTCom), 2010 International Conference on.

[49] Leistikow, R., \& Tavangarian, D. (2013, 25-28 March 2013). Secure Picture Data Partitioning for Cloud Computing Services. Paper presented at the Advanced Information Networking and
Applications Workshops (WAINA), 2013 27th International Conference on.

[50] Delettre, C., Boudaoud, K., \& Riveill, M. (2011, June 28 2011-July 1 2011). Cloud computing, security and data concealment. Paper presented at the Computers and Communications (ISCC), 2011 IEEE Symposium on.

[51] Mishra, R., Dash, S. K., Mishra, D. P., \& Tripathy, A. (2011, 8-10 April 2011). A privacy preserving repository for securing data across the cloud. Paper presented at the Electronics Computer Technology (ICECT), 2011 3rd International Conference on.

[52] Syam Kumar, P., Subramanian, R., \& Thamizh Selvam, D. (2010, 28-30 Oct. 2010). Ensuring data storage security in cloud computing using Sobol Sequence. Paper presented at the Parallel Distributed and Grid Computing (PDGC), 2010 1st International Conference on.

[53] Anane, R., Dhillon, S., \& Bordbar, B. (2008). Stateless data concealment for distributed systems. Journal of Computer and System Sciences, 74(2), 243-254. 